\title{
Differential Vertex Operations in Lagrangian Field Theory*
}

\author{
J. H. LOWENSTEIN \\ Department of Physics, University of Pittsburgh, Pittsburgh, Pennsylvania USA
}

Received July 5, 1971

\begin{abstract}
A general framework is derived for studying differential operations in renormalized perturbation theory. The method makes possible a simple, unified derivation of the renormalization group and Callan-Symanzik equations, as well as a direct test for broken symmetries (including broken scale invariance), without the necessity of defining currents and deriving their generalized Ward identites. A second-order differential equation of the Callan-Symanzik type is derived using similar methods.
\end{abstract}

\section{Introduction}

Various differential operations on the Green's functions of Lagrangian field theory have proven to be useful tools in investigating the renormalization properties [1,2] and short-distance behavior [3,4] of these functions. The aim of the present article is to formulate a simple general framework for studying differential operations (e.g. derivatives with respect to masses and coupling constants) within the context of $B P H Z$ $[1,5]$ renormalized perturbation theory (see [5] for further references). The method of differential vertex operations to be developed in Sections II through IV will make possible (a) a simple and unified derivation of the renormalization group $[6,1]$ and generalized Callan-Symanzik $[3,4]$ equations (Sections III B, C) (b) a method for verifying directly whether a given theory possesses broken symmetry (i. e. its truncated Green's functions are symmetric asymptotically for small distances), without introducing currents and generalized Ward identities (Section III D) and (c) the generalization of the Callan-Symanzik equations to second order, thus providing the basis a more detailed description of the asymptotic short-distance behavior of vertex functions (Section IV).

\section{Basic Concepts}

\section{A. Definition of Differential Vertex Operations}

Given a theory with basic fields $A^{(i)}(x)$ and effective Lagrangian

$$
\mathscr{L}_{\mathrm{EFF}}=\mathscr{L}_{0}+\mathscr{L}_{I}
$$

* Supported in part by the U. S. Atomic Energy Commission under Contract No. AT-30-1-3829.

1 Commun. math. Phys., Vol. 24 
as a functional of the $A^{(i)}$, the Gell-Mann-Low formula [7]

$$
\begin{aligned}
G_{i_{1} \ldots i_{N}}^{(N)}\left(x_{1}, x_{2}, \ldots, x_{N}\right) & \\
\equiv\left\langle 0\left|T \prod_{k} A^{\left(i_{k}\right)}\left(x_{k}\right)\right| 0\right\rangle= & \text { Finite part of }{ }^{(0)}\langle 0| T \prod_{k} A_{0}^{\left(i_{k}\right)}\left(x_{k}\right) \\
& \cdot e^{i \int: \mathscr{L}_{I}\left[A_{0}^{(i)]}\right] d^{4} x}|0\rangle^{(0)}
\end{aligned}
$$

where $A_{0}^{(i)}$ is the free field (specified by $\mathscr{L}_{0}$ ) corresponding to $A^{(i)}$ and the finite-part prescription is given by the $B P H Z[1,5]$ subtraction procedure, gives an unambiguous prescription for calculating the Green's functions (covariant time-ordered functions) of the theory to arbitrary order. We now wish to define, using Zimmermann's normal products [5], certain differential operations on the Green's functions. This will be accomplished by means of simple modifications of the Feynman rules specified by (2.1).

Suppose $P_{i}(x), i=1,2, \ldots$, is a covariant polynomial in the basic fields and their derivatives (usually contracted over all tensor and spinor indices) of dimension

$$
D_{i}=b_{i}+\frac{3}{2} f_{i}+d_{i}
$$

where $b_{i}$ and $f_{i}$ are the numbers of boson and fermion fields, respectively, and $d_{i}$ is the number of derivatives in $P_{i}(x)$. In the language of momentumspace Feynman diagrams, $P_{i}(x)$ corresponds to a vertex $V_{i}$ with $f_{i}$ fermion and $b_{i}$ boson legs, and includes a polynomial of degree $d_{i}$ in the momenta of those legs. The momentum $p_{i}$ entering at $V_{i}$ is the variable conjugate to $x$ under Fourier transformation, and clearly vanishes if $V_{i}$ is an internal vertex. Following Zimmermann [5], we define time-ordered functions involving normal products by means of the modified Gell-Mann-Low formula,

$$
\begin{aligned}
& \left\langle 0\left|T \prod_{j=1}^{M} N_{\delta_{j}}\left[P_{j}\left(y_{j}\right)\right] A^{\left(i_{1}\right)}\left(x_{1}\right) \ldots A^{\left(i_{N}\right)}\left(x_{N}\right)\right| 0\right\rangle \\
& =\text { Finite part of }{ }^{(0)}\langle 0| T \prod_{j=1}^{M}: P_{0 j}\left(y_{j}\right): A_{0}^{\left(i_{1}\right)}\left(x_{1}\right) \ldots A_{0}^{\left(i_{N}\right)}\left(x_{N}\right) \\
& \quad \cdot \exp \left\{i \int: \mathscr{L}_{I}\left[A_{0}^{(k)}\right]: d^{4} x\right\}|0\rangle^{(0)}
\end{aligned}
$$

where $A_{0}^{(i)}$ is the free field (specified by $\mathscr{L}_{0}$ ) corresponding to $A^{(i)}$ and $: P_{0 j}$ : is the Wick polynomial in the $A_{0}^{(i)}$ corresponding to $P_{j}$. The finitepart prescription is again that of $B P H Z$ [5], with the number of subtractions required for each proper subdiagram $\gamma$ governed by the degree function

$$
\delta(\gamma)=4-B-\frac{3}{2} F-\sum_{k}\left(4-\delta_{k}\right)
$$


where $B$ and $F$ are the numbers of external boson and fermion lines of $\gamma$, respectively, $\delta_{k}$ is the degree of vertex $V_{k}$ (an integer $\geqq$ the dimension $D_{k}$ ) and the summation is over all vertices, internal as well as external, of $\gamma$. For a renormalizable theory one can always choose $\delta_{k}=4$ for all vertices arising from the interaction Lagrangian in (2.2), in which case the summation in (2.3) would only involve the normal-product vertices.

In the present article we shall be principally concerned with differential vertex operations (DVO's) defined by

$$
\begin{aligned}
\prod_{i} \Delta_{V_{i}}^{\delta_{i}} G_{j_{1} \ldots j_{N}}^{(N)}\left(x_{1}, \ldots x_{N}\right) \\
\quad=\left\langle 0\left|T \prod_{i} \int d y_{i} N_{\delta_{t}}\left[P_{i}\left(y_{i}\right)\right] A^{\left(j_{1}\right)}\left(x_{1}\right) \ldots A^{\left(j_{N}\right)}\left(x_{N}\right)\right| 0\right\rangle .
\end{aligned}
$$

By keeping only the contributions of connected (resp. proper) diagrams to the righthand side of (2.4), one may equally well define $D V O$ 's on the truncated Green's (resp. vertex) functions, $G_{i_{1} \ldots i_{N}}^{(N)}\left(x_{1}, \ldots, x_{N}\right)^{T}$ (resp. $\left.\Gamma_{i_{1} \ldots i_{N}}^{(N)}\left(x_{1}, \ldots, x_{N}\right)\right)$. In the following we shall often use the symbol $F^{(N)}$ as a generic label for $G^{(N)}, G^{(N) T}$ and $\Gamma^{(N)}$.

\section{B. Differential Properties of DVO'S}

The differential character of the $D V O$ 's becomes evident of one examines the effect on the Green's functions of an infinitesimal change of the Lagrangian density

$$
\mathscr{L}_{I}(x) \rightarrow \mathscr{L}_{I}^{\varepsilon}(x)=\mathscr{L}_{I}(x)+\sum_{k} \varepsilon_{k} P_{k}(x)
$$

where the vertices $V_{k}$ corresponding to the scalar polynomials $P_{k}$ are assigned degrees $\delta_{k}$ in the $B P H Z$ subtraction procedure of the theory with modified Lagrangian. In zeroth order (in the $\varepsilon_{k}$ ), the Green's functions of the modified and unmodified Lagrangians coincide. The first-order contribution to $G_{i_{1} \ldots i_{N}}^{(N) \varepsilon}\left(x_{1}, \ldots x_{N}\right)$ is given by the sum of Feynman diagrams containing one and only one of the special vertices $V_{i}$. In terms of definition (2.4) this may be expressed as,

$$
\left.\frac{\partial G^{(N) \varepsilon}}{\partial \varepsilon^{k}}\right|_{\varepsilon_{j}=0, \text { all } j}=i \Delta_{V_{k}}^{\delta_{k}} G^{(N)}
$$

Extending the above reasoning to higher orders and to the other types of $N$-point functions yields

$$
\left.\frac{\partial^{m} F^{(N) \varepsilon}}{\partial \varepsilon_{k_{1}} \ldots \partial \varepsilon_{k_{m}}}\right|_{\varepsilon_{j}=0, \text { all j }}=i^{m} \Delta_{V_{k_{1}}}^{\delta_{k_{1}}} \ldots \Delta_{V_{k_{m}}}^{\delta_{k_{m}}} F^{(N)} .
$$


Of special importance is the case in which (2.5) arises from an infinitesimal increment in one of the parameters $g_{i}$ of the theory: $g_{i} \rightarrow g_{i}+\varepsilon$. Without loss of generality we may choose $\mathscr{L}_{0}^{\varepsilon}=\mathscr{L}_{0}$. Then, assuming analyticity of $\mathscr{L}$ in $g_{i}$,

$$
\mathscr{L}_{I} \rightarrow \mathscr{L}_{I}^{\varepsilon}=\mathscr{L}_{I}+\sum_{n=1}^{\infty} \frac{\varepsilon^{n}}{n !} \frac{\partial^{n} \mathscr{L}}{\partial g_{i}^{n}}
$$

If $\mathscr{L}(x)=\sum_{i=1}^{m} a_{i} Q_{i}(x)$, where the $Q_{i}$ are field products (corresponding to vertices $V_{i}$ ) and the $a_{i}$ are functions of the parameters $g_{j}$, then the same sort of reasoning which led to (2.6) gives us

$$
\begin{aligned}
\frac{\partial F^{(N)}}{\partial g_{k}} & =\sum_{i=1}^{m} \frac{\partial a_{i}}{\partial g_{k}} \Delta_{V_{i}}^{\delta_{i}} F^{(N)} \\
\frac{\partial}{\partial g_{k}}\left(\Delta_{V_{1}}^{\delta_{1}}\right)^{n_{1}} \ldots\left(\Delta_{V_{m}}^{\delta_{m}}\right)^{n_{m}} F^{(N)} & =\sum_{i=1}^{m} \frac{\partial a_{i}}{\partial g_{k}}\left(\Delta_{V_{1}}^{\delta_{1}}\right)^{n_{1}} \ldots\left(\Delta_{V_{l}}^{\delta_{2}}\right)^{n_{i}+1} \ldots\left(\Delta_{V_{m}}^{\delta_{m}}\right)^{n_{m}} F^{(N)} .
\end{aligned}
$$

Note that the vertices of $\mathscr{L}_{0}$ must be assigned degree 4 in order not to affect the number of $B P H Z$ subtractions. Formulas (2.8) may be used to calculate derivatives of arbitrary order with respect to the parameters $g_{i}$.

\section{Operational Properties of DVO's}

In what sense, if any, does $\prod_{i} \Delta_{V_{i}}^{\delta_{i}}$ operate on $F^{(N)}$ ? From the definition (2.4) alone the answer to this question is not obvious. Certainly a $D V O$ does not define a mapping on the space of tempered distributions $\mathscr{S}^{\prime}\left(R^{4 N}\right)$, or even on a special subspace. Actually, if one studies the method by which we defined $D V O$ 's, he will find that the latter act on the $F^{(N)}$ only via the Feynman rules used to construct these functions. Let us now make this notion more precise.

The set of free fields $A_{i}^{(0)}$, with propagators specified by $\mathscr{L}_{0}$, determines a set $\Sigma$ of possible Lorentz-invariant vertices $V$, each assigned an appropriate $B P H Z$ degree $\delta$. Let $\boldsymbol{V}$ be the vector space of all formal linear combinations of

and

$$
\left\{V_{1}, \ldots, V_{n}\right\}, \quad V_{k} \in \Sigma, \quad n=1,2, \ldots
$$

$$
\{\} \text { (notational convention: } n=0 \text { ). }
$$

The Abelian algebra of $D V O$ 's is represented as an algebra of linear operators in $\boldsymbol{V}$, with

$$
\prod_{i=1}^{l} \Delta_{W_{l}}^{\delta_{2}}\left\{V_{1}, \ldots, V_{n}\right\}=\left\{V_{1}, \ldots V_{n}, W_{1}, \ldots W_{l}\right\}, \quad l=1,2, \ldots, \quad n=0,1,
$$

and the action on other elements given by linearity. 
We now consider the linear mapping

$$
\phi_{F}^{(N)}: V \rightarrow \mathscr{S}^{\prime}\left(R^{4 N}\right)
$$

defined by

i) $\phi_{F}^{\left({ }^{(N)}\right)}(\{\})$ is the free $F^{(N)}$-function.

ii) If $\mathscr{V} \in V, \mathscr{V}=\left\{V_{1}, \ldots, V_{n}\right\}, n=1,2, \ldots$, then $\phi_{F}^{(N)}(\mathscr{V})$ is the $F^{(N)}$ function calculated using the available free propagators and the vertices $V_{1}, \ldots, V_{n}$, with the requirement that each diagram contain each $V_{k}$ once and only once. $\phi_{F}^{(N)}$ is defined on an arbitrary element of $\boldsymbol{V}$ by linearity. In this notation the Gell-Mann-Low formula (2.1) takes the form

$$
F^{(N)}=\Phi_{F}{ }^{(N)}\left(\exp \left[\sum_{i} \Delta_{U_{t}}^{\delta_{l}}\right]\{\}\right)
$$

where the $U_{i}$ are the interaction vertices, and (2.4) becomes

$$
\prod_{j} \Delta_{V_{j}}^{\delta_{j}} F^{(N)}=\Phi_{F}^{(N)}\left(\prod_{j} \Delta_{V_{j}}^{\delta_{j}} \exp \left[\sum_{i} \Delta_{U_{i}}^{\delta_{i}}\right]\{\}\right) .
$$

Eq. (2.10) and (2.11) give the precise formulation of the statement that $\Pi \Delta_{V_{j}}^{\delta_{j}} F^{(N)}$ is constructed by successive application of the operators $\Delta_{V_{j}}^{\delta_{j}}$ to the Feynman rules used to construct $F^{(N)}$.

\section{Counting Identities}

In Section II $b$ we saw that derivatives of Green's and vertex functions may conveniently be expressed in terms of differential vertex operations. In this section we shall see that the $D V O$ 's are equally useful in expressing the "counting identities" which are direct consequences of the "topological" structure of Feynman diagrams.

Let us suppose that the free propagators specified by $\mathscr{L}_{0}$ are classified into "types" labeled by an index $k=1,2, \ldots$ As far as the counting identities are concerned this classification may be any grouping of the free propagators into convenient subsets. If $U_{1}, \ldots, U_{m}$ are the interaction vertices corresponding to $i \mathscr{L}_{I}$, and $v_{k i}$ is the number of lines of type $k$ attached to a vertex of type $U_{i}$, then counting the number of line-ends of type $k$ in any diagram $\gamma$ gives

$$
N_{k}+2 n_{k}=\sum_{i}^{m} c_{i} v_{k i}
$$

where

$c_{i}=$ number of vertices of type $U_{i}$ in $\gamma$.

$N_{k}=$ number of external lines of $\gamma$ of type $k$.

$n_{k}=$ number of internal lines of $\gamma$ of type $k$. 
We now observe that $\Delta_{U_{j}}^{\delta_{j}}$ serves as a counting operator for interaction vertices of type $U_{j}$ :

$$
\begin{aligned}
\Delta_{U_{j}}^{\delta_{j}} \exp \left[\sum_{i=1}^{m} \Delta_{U_{i}}^{\delta_{1}}\right]\{\} & =\sum_{c_{i}=0}^{\infty} \frac{1}{c_{1} ! \ldots c_{m} !}\left(\Delta_{U_{1}}^{\delta_{1}}\right)^{c_{1}} \ldots\left(\Delta_{U_{j}}^{\delta_{j}}\right)^{c_{j}+1} \ldots\left(\Delta_{U_{m}}^{\delta_{m}}\right)^{c_{m}}\{\} \\
& =\sum_{c_{i}=0}^{\infty} \frac{c_{j}}{c_{1} ! \ldots c_{m} !}\left(\Delta_{U_{1}}^{\delta_{1}}\right)^{c_{1}} \ldots\left(\Delta_{U_{m}}^{\delta_{m}}\right)^{c_{m}}\{\} .
\end{aligned}
$$

Thus if

$$
F^{(N)}=\sum_{\gamma} F_{\gamma}^{(N)} \quad(\text { sum over Feynman diagrams })
$$

then

$$
\Delta_{U_{j}}^{\delta_{j}} F^{(N)}=\sum_{\gamma} c_{j}^{\gamma} F_{\gamma}^{(N)} .
$$

Similarly, if $W_{1}, \ldots, W_{l}$ are the 2-vertices corresponding to $i \mathscr{L}_{0}$ (assigned $B P H Z$ degree four), then

$$
\begin{aligned}
\Delta_{W_{k}}^{4} \Gamma^{(N)} & =-\sum_{\gamma} n_{k}^{\gamma} \Gamma^{(N)} \\
\Delta_{W_{k}}^{4} G^{(N)} & =-\sum_{\gamma}\left(n_{k}^{\gamma}+N_{k}\right) G^{(N)} \\
\Delta_{W_{k}}^{4} G^{(N) T} & =-\sum_{\gamma}\left(n_{k}^{\gamma}+N_{k}\right) G^{(N) T} .
\end{aligned}
$$

Eqs. (2.12), (2.13) and (2.14) may be combined to give us the identities

$$
\begin{aligned}
\left(N_{k}-2 \Delta_{W_{k}}^{4}\right) \Gamma^{(N)} & =\sum_{i=1}^{m} v_{k i} \Delta_{U_{i}}^{\delta_{i}} \Gamma^{(N)} \\
\left(-N_{k}-2 \Delta_{W_{k}}^{4}\right) G^{(N)} & =\sum_{i=1}^{m} v_{k i} \Delta_{U_{i}}^{\delta_{2}} G^{(N)} \\
\left(-N_{k}-2 \Delta_{W_{k}}^{4}\right) G^{(N) T} & =\sum_{i=1}^{m} v_{k i} \Delta_{U_{i}}^{\delta_{2}} G^{(N) T} .
\end{aligned}
$$

For $\prod_{i} \Delta_{V_{2}}^{\delta_{2}} F^{(N)}$ the topological relation becomes

$$
N_{k}+2 n_{k}=\sum_{i} c_{i} v_{k i}+\sum_{j} \mu_{k j}
$$

where $\mu_{k j}$ is the number of lines of type $k$ attached to a vertex of type $V_{j}$, and the counting identities (2.15) are modified by the addition of the terms $\sum_{j} \mu_{k j} \Delta_{V_{j}}^{\delta_{j}} F^{(N)}$ to the righthand members. 


\section{First Order Differential Operations}

\section{A. First Order Vertex Operations in the $A^{4}$ Model}

An excellent testing ground for the methods of differential vertex operations is provided by the $A^{4}$ model, whose effective Lagrangian is given [5] by

$$
\begin{aligned}
\mathscr{L}_{\mathrm{EFF}}= & \frac{1}{2} \partial_{\mu} A \partial^{\mu} A-\frac{1}{2} m^{2} A^{2}-\frac{g}{4 !} A^{4}+\frac{1}{2} a A^{2} \\
& +\frac{1}{2} b \partial_{\mu} A \partial^{\mu} A+\frac{1}{4 !} c A^{4}
\end{aligned}
$$

where $a, b$ and $c$ are power series in the coupling constant $g$ and are defined implicitly by the normalization conditions

$$
\begin{aligned}
& \left.\Gamma^{(2)}(p,-p)\right|_{p^{2}=m^{2}}=0 \\
& \left.\Gamma^{(2)}(p,-p)\right|_{p^{2}=\mu^{2}}=i\left(\mu^{2}-m^{2}\right) \\
& \left.\Gamma^{(4)}\left(p_{1}, p_{2}, p_{3}, p_{4}\right)\right|_{\text {symmetry point }}=-i g
\end{aligned}
$$

where

$$
(2 \pi)^{4} \delta\left(\sum_{i} p_{i}\right) \Gamma^{(N)}\left(p_{1}, \ldots, p_{N}\right)=\int d x_{1} \ldots \int d x_{N} \exp \left[i \sum_{k} p_{k} \cdot x_{k}\right] \Gamma^{(N)}\left(x_{1}, \ldots, x_{N}\right)
$$

and the symmetry point is defined by

$$
\begin{aligned}
p_{i}^{2} & =\mu^{2} & & i=1,2,3,4, \\
\left(p_{i}+p_{j}\right)^{2} & =\frac{4}{3} \mu^{2} & & i \neq j .
\end{aligned}
$$

In the $B P H Z$ subtraction procedure each of the interaction vertices is assigned degree four, so that the only renormalization parts are the self-energy diagrams with degree two and the four-vertex diagrams with degree zero. $\Gamma^{(2)}$ and $\Gamma^{(4)}$ have been normalized at $p^{2}=\mu^{2}$ rather than on the mass shell in order that we might be able to make finite renormalizations by changing the values of $\mu^{2}$ and $g$ with $m^{2}$ fixed (see Section III C).

The form of the effective Lagrangian (3.1) leads us to consider the following differential vertex operations of degree four:

$$
\begin{aligned}
& \Delta_{1}=\frac{i}{2} \int d^{4} x N_{4}\left[A^{2}(x)\right] \\
& \Delta_{2}=\frac{i}{2} \int d^{4} x N_{4}\left[\partial_{\mu} A(x) \partial^{\mu} A(x)\right] \\
& \Delta_{3}=\frac{i}{4 !} \int d^{4} x N_{4}\left[A^{4}(x)\right] .
\end{aligned}
$$


From (2.8), (2.15) and Zimmermann's formula [5] relating normal products of different degrees (see Appendix), one readily obtains the following identities:

$$
\begin{aligned}
\frac{\partial \Gamma^{(N)}}{\partial m^{2}} & =\left(\left(\frac{\partial a}{\partial m^{2}}-1\right) \Delta_{1}+\frac{\partial b}{\partial m^{2}} \Delta_{2}+\frac{\partial c}{\partial m^{2}} \Delta_{3}\right) \Gamma^{(N)}, \\
\frac{\partial \Gamma^{(N)}}{\partial \mu^{2}} & =\left(\frac{\partial a}{\partial \mu^{2}} \Delta_{1}+\frac{\partial b}{\partial \mu^{2}} \Delta_{2}+\frac{\partial c}{\partial \mu^{2}} \Delta_{3}\right) \Gamma^{(N)}, \\
\frac{\partial \Gamma^{(N)}}{\partial g} & =\left(\frac{\partial a}{\partial g} \Delta_{1}+\frac{\partial b}{\partial g} \Delta_{2}+\left(\frac{\partial c}{\partial g}-1\right) \Delta_{3}\right) \Gamma^{(N)}, \\
N \Gamma^{(N)} & =\left(2\left(a-m^{2}\right) \Delta_{1}+2(1+b) \Delta_{2}+4(c-g) \Delta_{3}\right) \Gamma^{(N)}, \\
\Delta_{0} \Gamma^{(N)} & =\left(\Delta_{1}+r \Delta_{2}+s \Delta_{3}\right) \Gamma^{(N)}
\end{aligned}
$$

where

$$
\begin{aligned}
\Delta_{0} & =\frac{i}{2} \int N_{2}\left[A^{2}(x)\right] d^{4} x, \\
r & =-i\left[\frac{d}{d p^{2}} \Delta_{0} \Gamma^{(2)}(p,-p)\right]_{p=0}, \\
s & =-i \Delta_{0} \Gamma^{(4)}(0,0,0,0) .
\end{aligned}
$$

We see that the five quantities $\frac{\partial \Gamma^{(N)}}{\partial m^{2}}, \frac{\partial \Gamma^{(N)}}{\partial \mu^{2}}, \frac{\partial \Gamma^{(N)}}{\partial g}, N \Gamma^{(N)}$ and $\Delta_{0} \Gamma^{(N)}$ are all linear combinations of the $\Delta_{i} \Gamma^{(N)} i=1,2,3$. Since the latter are linearly independent, it is clear that there will be two independent linear relations among the five operations. These may be conveniently chosen to be the generalized Callan-Symanzik equations and the renormalization group equations, to which we shall turn our attention in Sections III B and III C.

\section{B. Generalized Callan-Symanzik Equations}

From $(3.4 \mathrm{a}-\mathrm{e})$ it follows that the four operations $\left(m^{2} \frac{\partial}{\partial m^{2}}+\mu^{2} \frac{\partial}{\partial \mu^{2}}\right)$, $\frac{\partial}{\partial g}, N 1$ and $\Delta_{0}$ are linearly dependent. That is,

$$
\alpha m^{2} \Delta_{0} \Gamma^{(N)}=\left(m^{2} \frac{\partial}{\partial m^{2}}+\mu^{2} \frac{\partial}{\partial \mu^{2}}+\beta \frac{\partial}{\partial g}-N \gamma\right) \Gamma^{(N)}
$$

where $\alpha, \beta$ and $\gamma$ are, on dimensional grounds, functions of $g$ and the ratio $\mathrm{m}^{2} / \mu^{2}$. To solve for these quantities, we equate coefficients of the basic $D V O$ 's $\Delta_{i}, i=1,2,3$, making use of the scaling equations (ordinary 
dimensional analysis):

$$
\begin{aligned}
& m^{2} \frac{\partial a}{\partial m^{2}}+\mu^{2} \frac{\partial a}{\partial \mu^{2}}=a \\
& m^{2} \frac{\partial b}{\partial m^{2}}+\mu^{2} \frac{\partial b}{\partial \mu^{2}}=0=m^{2} \frac{\partial c}{\partial m^{2}}+\mu^{2} \frac{\partial c}{\partial \mu^{2}} .
\end{aligned}
$$

Then

$$
\begin{aligned}
\alpha m^{2} & =a-m^{2}+\frac{\partial a}{\partial g} \beta-2\left(a-m^{2}\right) \gamma \\
\alpha m^{2} r & =\frac{\partial b}{\partial g} \beta-2(1-b) \gamma \\
\alpha m^{2} s & =\left(\frac{\partial a}{\partial g}-1\right) \beta-4(c-g) \gamma .
\end{aligned}
$$

Since the determinant of the coefficients of $\alpha, \beta$ and $\gamma$ is nonzero in zeroth order, a solution always exists in perturbation theory. Eq. (3.5) is a generalization of the Callan-Symanzik equations, which correspond to the case $\mu^{2}=m^{2}$.

More concise expressions for the coefficients may be obtained by employing the normalization conditions (3.2) at $\mathrm{m}^{2}$ and at $\mu^{2}$. For the latter it is convenient to introduce (notation of [2])

and

$$
d\left(\frac{p^{2}}{\mu^{2}}, \frac{m^{2}}{\mu^{2}}, g\right)=i\left(p^{2}-m^{2}\right)\left[\Gamma^{(2)}(p,-p)\right]^{-1}
$$

$$
q\left(\frac{p^{2}}{\mu^{2}}, \frac{m^{2}}{\mu^{2}}, g\right)=\left.\mathrm{id}\left(\frac{p^{2}}{\mu^{2}}, \frac{m^{2}}{\mu^{2}}, g\right)^{2} \Gamma^{(4)}\left(p_{1}, p_{2}, p_{3}, p_{4}\right)\right|_{\text {sym.pt. }\left(p^{2}\right)}
$$

which satisfy

$$
\begin{aligned}
& d\left(1, \frac{m^{2}}{\mu^{2}}, g\right)=1 \\
& q\left(1, \frac{m^{2}}{\mu^{2}}, g\right)=g
\end{aligned}
$$

and, from (3.5)

$$
\begin{aligned}
\left(m^{2} \frac{\partial}{\partial m^{2}}+\mu^{2} \frac{\partial}{\partial \mu^{2}}+\beta \frac{\partial}{\partial g}+2 \gamma\right) d & =\xi d \\
\left(m^{2} \frac{\partial}{\partial m^{2}}+\mu^{2} \frac{\partial}{\partial \mu^{2}}+\beta \frac{\partial}{\partial q}\right) q & =n q
\end{aligned}
$$


where

$\xi\left(\frac{p^{2}}{\mu^{2}}, \frac{m^{2}}{\mu^{2}}, q\right)=-m^{2}\left[\frac{\alpha \Delta_{0} \Gamma^{(2)}(p,-p)}{\Gamma^{(2)}(p,-p)}+\frac{1}{p^{2}-m^{2}}\right]$, $\eta\left(\frac{p^{2}}{\mu^{2}}, \frac{m^{2}}{\mu^{2}}, g\right)=\frac{\left.\alpha m^{2} \Delta_{0} \Gamma^{(4)}\left(p_{1}, p_{2}, p_{3}, p_{4}\right)\right|_{\text {sym.pt. }\left(p^{2}\right)}}{\left.\Gamma^{(4)}\left(p_{1}, p_{2}, p_{3}, p_{4}\right)\right|_{\text {sym.pt. }\left(p^{2}\right)}}+2 \xi\left(\frac{p^{2}}{\mu^{2}}, \frac{m^{2}}{\mu^{2}}, g\right)$.

From (3.10) evaluated at $p^{2}=\mu^{2}$, we get

$$
\begin{aligned}
2 \gamma\left(\frac{m^{2}}{\mu^{2}}, g\right) & =d_{1}\left(1, \frac{m^{2}}{\mu^{2}}, g\right)+\xi\left(1, \frac{m^{2}}{\mu^{2}}, g\right) \\
\beta\left(\frac{m^{2}}{\mu^{2}}, g\right) & =q_{1}\left(1, \frac{m^{2}}{\mu^{2}}, g\right)+g \eta\left(1, \frac{m^{2}}{\mu^{2}}, g\right),
\end{aligned}
$$

where the index " 1 " indicates differentiation with respect to the first argument. Eq. (3.5) for $N=2$, evaluated at $p^{2}=m^{2}$, then yields

$$
\alpha\left(\frac{m^{2}}{\mu^{2}}, g\right)=\left[-\left.i m^{2} \Delta_{0} \Gamma^{(2)}(p,-p)\right|_{p^{2}=m^{2}} d\left(\frac{m^{2}}{\mu^{2}}, \frac{m^{2}}{\mu^{2}}, g\right)\right]^{-1} .
$$

The reader is referred to Refs. [3, 4] for discussions of how Eq. (3.5) may be used to describe the short-distance behavior of vertex functions. The crucial point is that for $p_{i}=\lambda r_{i}, \lambda \rightarrow \infty$ (a certain set of measure zero excluded) the ratio

$$
\frac{\Delta_{0} \Gamma^{(N)}\left(p_{1}, \ldots, p_{N}\right)}{\Gamma^{(N)}\left(p_{1}, \ldots, p_{N}\right)}
$$

can be shown by power-counting arguments to vanish like $\lambda^{-2}$ (up to logarithms), and thus the asymptotic vertex functions satisfy (3.5) with the lefthand side set equal to zero. More delicate restrictions on the asymptotic behavior will be discussed in Section IV with the aid of second-order $D V O$ 's.

\section{Renormalization Group Equations}

The identities $(3.4 \mathrm{a}-\mathrm{e})$ will now be used to derive the following differential equations for the vertex functions of the $A^{4}$ theory,

$$
\left(\mu^{2} \frac{\partial}{\partial \mu^{2}}+\sigma\left(\frac{m^{2}}{\mu^{2}}, g\right) \frac{\partial}{\partial g}-N \tau\left(\frac{m^{2}}{\mu^{2}}, g\right)\right) \Gamma^{(N)}\left(p_{1}, \ldots ; p_{N} ; m^{2}, \mu^{2}, g\right)=0
$$

where the dependence of $\Gamma^{(N)}$ on all parameters has been displayed explicitly. The one-dimensional Lie group of transformations on the 
half-plane $\left\{\left(\mu^{2}, g\right): \mu^{2}>0\right\}$ generated by

$$
\mu^{2} \frac{\partial}{\partial \mu^{2}}+\sigma\left(\frac{m^{2}}{\mu^{2}}, g\right) \frac{\partial}{\partial g}
$$

is the renormalization group $[1,6]$, and (3.12) expresses the invariance property of the vertex functions with respect to this group. We see that if the normalization mass $\mu^{2}$ undergoes an infinitesimal dilation, $\mu^{2} \rightarrow \mu^{2}(1+\varepsilon)$, and the coupling constant $g$ is changed at the same time to $g+\varepsilon \sigma\left(\frac{m^{2}}{\mu^{2}}, g\right)$, then the effect on the vertex (and Green's) functions is the same as if we had simply multiplied each field by the infinitesimal " $Z$ factor", $1-\varepsilon \tau\left(\frac{m^{2}}{\mu^{2}}, g\right)$.

Referring to $(3.4 \mathrm{~b}, \mathrm{c}, \mathrm{d})$, we see that in order to satisfy (3.12) it must be possible to select $\sigma$ and $\tau$ in such a way that

$$
\begin{aligned}
& \mu^{2} \frac{\partial a}{\partial \mu^{2}}+\frac{\partial a}{\partial g} \sigma-2\left(a-m^{2}\right) \tau=0, \\
& \mu^{2} \frac{\partial b}{\partial \mu^{2}}+\frac{\partial b}{\partial g} \sigma-2(1+b) \tau=0, \\
& \mu^{2} \frac{\partial c}{\partial \mu^{2}}+\left(\frac{\partial c}{\partial g}-1\right) \sigma-4(c-g) \tau=0,
\end{aligned}
$$

are fulfilled. The crucial point in verifying (3.13) is that none of the operations $\frac{\partial}{\partial \mu^{2}}, \frac{\partial}{\partial g}, 1$ changes the position of the propagator pole at $p^{2}=m^{2}$. Hence

$$
\left[\left(\mu^{2} \frac{\partial}{\partial \mu^{2}}+\sigma \frac{\partial}{\partial g}-2 \tau\right) \Gamma^{(2)}(p,-p)\right]_{p^{2}=m^{2}}=0 .
$$

Once we have chosen $\sigma$ and $\tau$ to satisfy (3.13b) and (3.13c) - which is always possible, thanks to the nonvanishing of the determinent of coefficients - we may use $(3.4 \mathrm{~b}-\mathrm{d})$ to rewrite $(3.14)$ as

$$
\left.\left(\mu^{2} \frac{\partial a}{\partial \mu^{2}}+\sigma \frac{\partial a}{\partial g}-2 \tau\left(a-m^{2}\right)\right) \Delta_{1} \Gamma^{(2)}(p,-p)\right|_{p^{2}=m^{2}}=0 .
$$

The trivial nonvanishing of $\Delta_{1} \Gamma^{(2)}$ in zeroth order then gives (3.13a).

Before proceeding to the more familiar integrated form of the renormalization group equations, let us use the normalization conditions at $\mu^{2}$ to derive convenient expressions for $\sigma$ and $\tau$. Applying (3.12) to the auxiliary functions $d\left(\frac{p^{2}}{\mu^{2}}, \frac{m^{2}}{\mu^{2}}, g\right)$ and $q\left(\frac{p^{2}}{\mu^{2}}, \frac{m^{2}}{\mu^{2}}, g\right)$ defined in (3.8), 
one readily verifies

$$
\begin{aligned}
\left(\mu^{2} \frac{\partial}{\partial \mu^{2}}+\sigma \frac{\partial}{\partial g}+2 \tau\right) d & =0 \\
\left(\mu^{2} \frac{\partial}{\partial \mu^{2}}+\sigma \frac{\partial}{\partial g}\right) q & =0 .
\end{aligned}
$$

Note that $q\left(\frac{p^{2}}{\mu^{2}}, \frac{m^{2}}{\mu^{2}}, g\right)$ is invariant under the renormalization group transformations (it is sometimes referred to as the invariant charge $[1,2]$ ). Evaluating (3.16) at $\mu^{2}=p^{2}$ and applying (3.8) then yield

$$
\begin{aligned}
\sigma\left(\frac{m^{2}}{\mu^{2}}, g\right) & =q_{1}\left(1, \frac{m^{2}}{\mu^{2}}, g\right) \\
2 \tau\left(\frac{m^{2}}{\mu^{2}}, g\right) & =d_{1}\left(1, \frac{m^{2}}{\mu^{2}}, g\right)
\end{aligned}
$$

where the index " 1 " indicates differentiation with respect to the first argument.

Referring back to $(3.11 \mathrm{a}, \mathrm{b})$, we see that the Callan-Symanzik and renormalization group coefficients are related by

$$
\begin{aligned}
& \beta\left(\frac{m^{2}}{\mu^{2}}, g\right)=\sigma\left(\frac{m^{2}}{\mu^{2}}, g\right)+g \eta\left(1, \frac{m^{2}}{\mu^{2}}, g\right) \\
& \gamma\left(\frac{m^{2}}{\mu^{2}}, g\right)=\tau\left(\frac{m^{2}}{\mu^{2}}, g\right)+\frac{1}{2} \xi\left(1, \frac{m^{2}}{\mu^{2}}, g\right) .
\end{aligned}
$$

For $m \rightarrow 0$ the coefficients are expected, by the arguments of Gell-Mann and Low [6], to approach finite limits. In this case both $\xi$ and $\eta$ vanish and we obtain, as expected,

$$
\begin{aligned}
& \beta(0, g)=\sigma(0, g) \\
& \gamma(0, g)=\tau(0, g) .
\end{aligned}
$$

The differential Eq. (3.16) for $q\left(\frac{p^{2}}{\mu^{2}}, \frac{m^{2}}{\mu^{2}}, g\right)$ may be readily integrated using standard mathematical methods [8]. One considers the oneparametric family of curves $\mathscr{C}$ (characteristic curves) in the halfplane $\left\{\left(\mu^{2}, g\right): \mu^{2}>0\right.$ with the property that a characteristic curve passing through the point $\left(\mu^{2}, g\right)$ has an unnormalized tangent vector $\left(\mu^{2}, \beta\right)$. Clearly one and only one curve in $\mathscr{C}$ passes through each point of the half-plane. From (3.16) and (3.18) it is clear that $q\left(\frac{p^{2}}{\mu^{2}}, \frac{m^{2}}{\mu^{2}}, g\right)$ is constant along each characteristic curve $C$, with its value determined by the 
intercept of $C$ with $\mu^{2}=p^{2}$ if $p^{2}>0$. Thus, for $p^{2}>0, \mu^{2}>0, \mu^{\prime 2}>0$ and all $g$,

$$
q\left(\frac{p^{2}}{\mu^{2}}, \frac{m^{2}}{\mu^{2}}, g\right)=q\left(\frac{p^{2}}{\mu^{\prime 2}}, \frac{m^{2}}{\mu^{\prime 2}}, q\left(\frac{\mu^{\prime 2}}{\mu^{2}}, \frac{m^{2}}{\mu^{2}}, g\right)\right) .
$$

Only continuous differentiability of $q\left(\frac{p^{2}}{\mu^{2}}, \frac{m^{2}}{\mu^{2}}, g\right)$ was assumed in the derivation of (3.20), but we must resort to its full analytic properties in order to extend this equation to include negative values of $p^{2}$. Eq. (3.20), thus extended, is the traditional form of the renormalization group equation for the invariant charge $q$.

To obtain the global equations for $d\left(\frac{p^{2}}{\mu^{2}}, \frac{m^{2}}{\mu^{2}}, g\right)$ and $\Gamma^{(N)}\left(p_{1}, \ldots p_{N} ; m^{2}, \mu^{2}, g\right)$ it is convenient to make a change of variables,

$$
\left(\mu^{2}, g\right) \rightarrow\left(\mu^{2}, q\right)
$$

with $\mu^{2}>0$ and $m^{2}$ and all momentum variables fixed. This is permissible, since the Jacobian

$$
J=\frac{\partial q}{\partial g}
$$

can vanish only at a point which is traversed by more than one characteristic curve, i.e. only where $\mu^{2}=0=\sigma$. In terms of the new variables, Eq. (3.12) and (3.16) take the form

$$
\begin{aligned}
& \mu^{2} \frac{\partial \hat{d}}{\partial \mu^{2}}=-2 \tau\left(\frac{m^{2}}{\mu^{2}}, \hat{g}\right) \hat{d} \\
& \mu^{2} \frac{\partial \hat{\Gamma}}{\partial \mu^{2}}=N \tau\left(\frac{m^{2}}{\mu^{2}}, \hat{g}\right) \hat{\Gamma}
\end{aligned}
$$

where

$$
\begin{gathered}
\hat{d}\left(p^{2} ; m^{2}, \mu^{2}, q\right)=d\left(\frac{p^{2}}{\mu^{2}}, \frac{m^{2}}{\mu^{2}}, g\right), \\
\hat{\Gamma}^{(N)}\left(p_{1} \ldots p_{N} ; m^{2}, \mu^{2}, q\right)=\Gamma^{(N)}\left(p_{1} \ldots p_{N} ; m^{2}, \mu^{2}, g\right), \\
\hat{g}\left(p^{2}, m^{2}, \mu^{2}, q\right)=g=q\left(\frac{\mu^{2}}{p^{2}}, \frac{m^{2}}{p^{2}}, q\right) .
\end{gathered}
$$

These may be integrated directly and exponentiated, giving

$$
\begin{aligned}
\hat{d}\left(p^{2} ; m^{2}, \mu^{2}, q\right) & =\exp \left[2 \int_{\mu^{2}}^{p^{2}} \frac{d \mu^{\prime 2}}{\mu^{\prime 2}}\left(\frac{m^{2}}{\mu^{\prime 2}}, q\left(\frac{\mu^{\prime 2}}{p^{2}}, \frac{m^{2}}{p^{2}}, q\right)\right)\right] \\
\hat{\Gamma}^{(N)}\left(p_{1}, \ldots, p_{N} ; m^{2}, \mu^{2}, q\right) & =\hat{\Gamma}^{(N)}\left(p_{1}, \ldots, p_{N} ; m^{2}, p^{2}, q\right) \hat{d}\left(p^{2}, m^{2}, \mu^{2}, q\right)^{-N / 2}
\end{aligned}
$$


from which follow, using (3.20), the global renormalization group equations,

$$
\begin{aligned}
d\left(\frac{p^{2}}{\mu^{2}}, \frac{m^{2}}{\mu^{2}}, g\right)= & d\left(\frac{\mu^{\prime 2}}{\mu^{2}}, \frac{m^{2}}{\mu^{2}}, g\right) d\left(\frac{p^{2}}{\mu^{\prime 2}}, \frac{m^{2}}{\mu^{\prime 2}}, q\left(\frac{\mu^{\prime 2}}{\mu^{2}}, \frac{m^{2}}{\mu^{2}}, g\right)\right) \\
\Gamma^{(N)}\left(p_{1}, \ldots, p_{N}, m^{2}, \mu^{2}, g\right)= & \Gamma^{(N)}\left(p_{1}, \ldots, p_{N} ; m^{2}, \mu^{\prime 2}, q\left(\frac{\mu^{\prime 2}}{\mu^{2}}, \frac{m^{2}}{\mu^{2}}, g\right)\right) \\
& \cdot d\left(\frac{\mu^{\prime 2}}{\mu^{2}}, \frac{m^{2}}{\mu^{2}}, g\right)^{-N / 2} .
\end{aligned}
$$

As pointed out by Gell-Mann and Low [6], the renormalization group equations for the theory with $m=0$ give some information concerning the asymptotic behavior of vertex functions of the theory with $m \neq 0$ and $\mu^{2}=m^{2}$. It should be noted, however, that the asymptotic region is not (unless one is lucky) the same as that where the homogeneous CallanSymanzik equations are applicable, namely where all momenta become uniformly large with the coupling constant fixed; rather it is where the momenta become large with the invariant charge fixed. Specifically, if $p=\lambda r, p_{i}=\lambda r_{i}$ with $\lambda \rightarrow \infty$ and $q$ fixed, then (3.22) gives

$$
\begin{gathered}
\left(-p^{2} \frac{\partial}{\partial p^{2}}+\beta(0, q) \frac{\partial}{\partial q}+\lambda \tau(0, q)\right) \hat{d}\left(p^{2} ; m^{2}, m^{2}, q\right) \sim 0 \\
\hat{\Gamma}^{(N)}\left(p_{1}, \ldots, p_{N} ; m^{2}, m^{2}, q\right) \hat{d}\left(p^{2} ; m^{2}, m^{2}, q\right)^{N / 2} \sim \hat{\Gamma}\left(p_{1}, \ldots, p_{N} ; 0, p^{2}, q\right) .
\end{gathered}
$$

From the work of Gell-Mann and Low [6] one expects that the indicated passage to the zero-mass limit may be performed without encountering divergences.

\section{Testing for Broken Symmetry}

A theory is said to possess a broken symmetry if its Green's (or vertex) functions become asymptotically invariant under a certain group of transformations in the short-distance limit (in momentum space, $\left.p_{i}=\lambda r_{i}, \lambda \rightarrow \infty\right)[9,10]$. The use of first-order $D V O$ 's provides the most direct means of testing a given Lagrangian model for the presence of broken symmetries. The principal advantage of the method is that it avoids the necessity of explicitly specifying a current and of deriving a generalized Ward identity for that current (compare with [9]).

The first application of our method will be to the investigation of broken scale invariance in the $A^{4}$ model. The starting point is the scaling equation

$$
\left[\frac{1}{2} \sum_{i=1}^{N-1} p_{i}^{\mu} \frac{\partial}{\partial p_{i}^{\mu}}+m^{2} \frac{\partial}{\partial m^{2}}+\mu^{2} \frac{\partial}{\partial \mu^{2}}-\frac{1}{2}(4-N)\right] \Gamma^{(N)}\left(p_{1}, \ldots, p_{N}\right)=0,
$$


which, using (3.4) and (3.6), may be written as

$$
\begin{aligned}
{\left[\sum_{i=1}^{N-1} p_{i}^{\mu}\right.} & \left.\frac{\partial}{\partial p_{i}^{\mu}}-4+N d\right] \Gamma^{(N)}\left(p_{1}, \ldots, p_{N}\right) \\
= & {\left[2\left(m^{2}-a\right) \Delta_{1}+(d-1)\left(2\left(a-m^{2}\right) \Delta_{1}+2(1+b) \Delta_{2}+4(c-g) \Delta_{3}\right)\right] } \\
& \cdot \Gamma^{(N)}\left(p_{1}, \ldots, p_{N}\right) .
\end{aligned}
$$

The parameter $d$ has been inserted to allow for anomalous Wilson dimension [11]. For broken scale invariance the three $D V O$ 's of degree four would have to conspire to give a righthand side which becomes neglible in the asymptotic region. The only known way of doing this is via (3.4e). However, a simple second-order calculation [9] shows this to be impossible: the two parameters available (Wilson dimension and normalization) are simply not sufficient to fix the three coefficients of (3.4e).

As a second example we consider the model of a two-component scalar field with effective Lagrangian

$$
\begin{aligned}
\mathscr{L}_{\mathrm{EFF}}= & \frac{1}{2} \partial_{\mu} A_{i} \partial^{\mu} A_{i}-\frac{1}{2} m_{1}^{2} A_{1}^{2}-\frac{1}{2} m_{2}^{2} A_{2}^{2}-\frac{g}{8}\left(A_{i} A_{i}\right)^{2} \\
& +\frac{1}{2} a_{1} A_{1}^{2}+\frac{1}{2} a_{2} A^{2}+\frac{1}{2} Z_{i j} \partial_{\mu} A_{i} \partial^{\mu} A_{j}-\frac{1}{4 !} g_{i j k l} A_{i} A_{j} A_{k} A_{l},
\end{aligned}
$$

where $g, m_{1}$ and $m_{2}$ are given and the other coefficients (power series in $g$ ) are to be adjusted to fix various normalization conditions and the requirement of asymptotic orthogonal symmetry (compare Section V of [9]).

In order to study the effect of " $i$-spin" rotations on the truncated Green's functions, we start with a symmetric free Lagrangian, writing

$$
\begin{aligned}
\mathscr{L}_{0}= & \frac{1}{2} \partial_{\mu} A_{i} \partial^{\mu} A_{i}-\frac{1}{2} m^{2} A_{i} A_{i} \\
\mathscr{L}_{I}= & -\frac{1}{2} m_{i j} A_{i} A_{j}-\frac{g}{8}\left(A_{i} A_{i}\right)^{2}+\frac{1}{2} Z_{i j} \partial_{\mu} A_{i} \partial^{\mu} A_{j} \\
& -\frac{1}{4 !} g_{i j k l} A_{i} A_{j} A_{k} A_{l}
\end{aligned}
$$

where $m^{2}$ is an average of $m_{1}^{2}$ and $m_{2}^{2}$ whose precise choice is not critical. Then, by the Gell-Mann-Low formula (2.1) and the $i$-spin invariance of the free vacuum, the effects of an $i$-spin rotation

$$
R_{i j}(\theta)=\left(\begin{array}{rr}
\cos \theta & \sin \theta \\
-\sin \theta & \cos \theta
\end{array}\right)
$$


can be thrown over onto $\mathscr{L}_{I}$ :

$$
\begin{aligned}
& \prod_{k=1}^{N} R_{i k j k} G_{j_{1} \ldots j_{N}}\left(x_{1} \ldots x_{N}\right) \\
& \quad=\text { Finite part of }{ }^{(0)}\left\langle 0\left|T A_{i_{1}}\left(x_{1}\right) \ldots A_{i_{N}}\left(x_{N}\right) \exp \left[i \int: \mathscr{L}_{I}^{\theta}: d^{4} x\right]\right| 0\right\rangle
\end{aligned}
$$

where

$$
\begin{aligned}
\mathscr{L}_{I}^{\theta}= & -\frac{g}{8}\left(A_{i} A_{i}\right)^{2}-\frac{1}{2} m_{i j}(\theta) A_{i} A_{j}+\frac{1}{2} Z_{i j}(\theta) \partial_{\mu} A_{i} \partial^{\mu} A_{j} \\
& -\frac{1}{4 !} g_{i j k l}(\theta) A_{i} A_{j} A_{k} A_{l} \\
m_{i j}(\theta)= & R_{i k}(\theta) R_{j l}(\theta) m_{k l}, \\
Z_{i j}(\theta)= & R_{i k}(\theta) R_{j l}(\theta) Z_{k l}, \\
g_{i j k l}(\theta)= & R_{i m}(\theta) R_{j n}(\theta) R_{k p}(\theta) R_{l q}(\theta) g_{m n p q} .
\end{aligned}
$$

The structure of each Feynman diagram contributing to the GellMann-Low formula is unchanged by the rotation, so that the $B P H Z$ subtraction procedure defining the finite part in (3.29) is unaltered.

Differentiating (3.29) with respect to $\theta$ at $\theta=0$ and applying (2.8), we obtain

$$
\begin{aligned}
\sum_{k=1}^{N} \tau_{i k j} G_{i_{1}}^{(N)} \ldots j \ldots i_{N} & \left(x_{1}, \ldots, x_{k}, \ldots, x_{N}\right)^{T} \\
& =\left(-m_{i j}^{\prime} \Delta_{l i j}+Z_{i j}^{\prime} \Delta_{2 i j}-g_{i j k l}^{\prime} \Delta_{3 i j k l}\right) G^{(N)}\left(x_{1}, \ldots, x_{N}\right)^{T}
\end{aligned}
$$

where

$$
\begin{aligned}
\tau_{i j} & =\left.\frac{d}{d \theta} R(\theta)\right|_{\theta=0}, \quad m_{i j}^{\prime}=\left.\frac{d}{d \theta} m_{i j}(\theta)\right|_{\theta=0}, \quad \text { etc. } \\
\Delta_{l i j} & =\frac{i}{2} \int N_{4}\left[A_{i}(x) A_{j}(x)\right] d^{4} x, \\
\Delta_{2 i j} & =\frac{i}{2} \int N_{4}\left[\partial_{\mu} A_{i}(x) \partial^{\mu} A_{j}(x)\right] d^{4} x, \\
\Delta_{3 i j k l} & =\frac{i}{4 !} \int N_{4}\left[A_{i}(x) A_{j}(x) A_{k}(x) A_{l}(x)\right] d^{4} x .
\end{aligned}
$$

This is precisely the "integrated Ward identity" derived in with the aid of a "broken-symmetry current" in Ref. [9]. The condition of broken invariance under $i$-spin rotations is that the righthand side of (3.30) be "soft" in the short-distance region. In Ref. [9] it is shown that this may be accomplished by so choosing the $m_{i j}, z_{i j}$ and $g_{i j k l}$ that the 
righthand side of (3.30) becomes proportional to $\Delta_{0 i j} G^{(N) T}$, where

$$
\Delta_{0 i j}=\frac{i}{2} \int N_{2}\left[A_{i}(x) A_{j}(x)\right] d^{4} x .
$$

The method employed in this example can obviously be applied to any perturbative model which one wishes to test for broken internal symmetry.

\section{Higher Order Differential Operations in the $A^{4}$ Model}

Eqs. (3.4) are easily generalized to apply to arbitrary $\Gamma_{n_{1}, n_{2}, n_{3}}^{(N)}=\Delta_{1}^{n_{1}} \Delta_{2}^{n_{2}} \Delta_{3}^{n_{3}} \Gamma^{(N)}$ :

$$
\begin{gathered}
\left(m^{2} \frac{\partial}{\partial m^{2}}+\mu^{2} \frac{\partial}{\partial \mu^{2}}\right) \Gamma_{n_{1}, n_{2}, n_{3}}^{(N)}=\left(a-m^{2}\right) \Gamma_{n_{1}+1, n_{2}, n_{3}}, \\
\frac{\partial}{\partial g} \Gamma_{n_{1}, n_{2}, n_{3}}^{(N)}=\left(\frac{\partial a}{\partial g} \Delta_{1}+\frac{\partial b}{\partial g} \Delta_{2}+\left(\frac{\partial c}{\partial g}-1\right) \Delta_{3}\right) \Gamma_{n_{1}, n_{2}, n_{3}}^{(N)}, \\
\left(N-2 n_{1}-2 n_{2}-4 n_{3}\right) \Gamma_{n_{1}, n_{2}, n_{3}}^{(N)} \\
=\left(2\left(a-m^{2}\right) \Delta_{1}+2(1+b) \Delta_{2}+4(c-g) \Delta_{3}\right) \Gamma_{n_{1}, n_{2}, n_{3}}^{(N)}, \\
\frac{\partial}{\partial \mu^{2}} \Gamma_{n_{1}, n_{2}, n_{3}}^{(N)}=\left(\frac{\partial a}{\partial \mu^{2}} \Delta_{1}+\frac{\partial b}{\partial \mu^{2}} \Delta_{2}+\frac{\partial c}{\partial \mu^{2}} \Delta_{3}\right) \Gamma_{n_{1}, n_{2}, n_{3}}^{(N)} .
\end{gathered}
$$

One readily verifies that the determinant of the coefficients of $\Gamma_{n_{1}+1, n_{2}, n_{3}}^{(N)}$, $\Gamma_{n_{1}, n_{2}+1, n_{3}}^{(N)}$ and $\Gamma_{n_{1}, n_{2}, n_{3}+1}^{(N)}$ in the righthand members of $(4.1 \mathrm{a}-\mathrm{c})$ is nonzero in zeroth order, so that these equations may be used recursively to write

$$
\Gamma_{n_{1}, n_{2}, n_{3}}^{(N)}=\mathscr{P}_{n_{1}, n_{2}, n_{3}}\left(m^{2} \frac{\partial}{\partial m^{2}}+\mu^{2} \frac{\partial}{\partial \mu^{2}}, \frac{\partial}{\partial g}\right) \Gamma^{(N)}
$$

where $\mathscr{P}_{n_{1}, n_{2}, n_{3}}$ is a polynomial of degree $n_{1}+n_{2}+n_{3}$.

From Eqs. (4.1), (3.7) and (3.13) follow immediately the renormalization group and generalized Callan-Symanzik equations for the $\Gamma_{n_{1}, n_{2}, n_{3}}^{(N)}$

$$
\begin{aligned}
& \left(\mu^{2} \frac{\partial}{\partial \mu^{2}}+\sigma \frac{\partial}{\partial g}-\left(N-2 n_{1}-2 n_{2}-4 n_{3}\right) \tau\right) \Gamma_{n_{1}, n_{2}, n_{3}}^{(N)}=0 \\
& \left(m^{2} \frac{\partial}{\partial m^{2}}+\mu^{2} \frac{\partial}{\partial \mu^{2}}+\beta \frac{\partial}{\partial g}-\left(N-2 n_{1}-2 n_{2}-4 n_{3}\right) \gamma\right) \Gamma_{n_{1}, n_{2}, n_{3}}^{(N)} \\
& =\alpha m^{2}\left(\Delta_{1}+r \Delta_{2}+s \Delta_{3}\right) \Gamma_{n_{1}, n_{2}, n_{3}}^{(N)} .
\end{aligned}
$$


As a final application of $D V O$ 's, let us derive a second-order refinement of the generalized Callan-Symanzik equations. From (4.3) we have

$$
\begin{aligned}
& {\left[m^{2} \frac{\partial}{\partial m^{2}}+\mu^{2} \frac{\partial}{\partial \mu^{2}}+\beta \frac{\partial}{\partial g}-(N-2) \gamma\right] \Delta_{0} \Gamma^{(N)}} \\
& =\alpha m^{2}\left(\Delta_{1}+r \Delta_{2}+s \Delta_{3}\right)^{2} \Gamma^{(N)}+\left(m^{2} \frac{\partial r}{\partial m^{2}}+\mu^{2} \frac{\partial r}{\partial \mu^{2}}+\beta \frac{\partial r}{\partial g}\right) \Delta_{2} \Gamma^{(N)} \\
& \quad+\left(m^{2} \frac{\partial s}{\partial m^{2}}+\mu^{2} \frac{\partial s}{\partial \mu^{2}}+\beta \frac{\partial s}{\partial g}-2 \gamma s\right) \Delta_{3} \Gamma^{(N)}
\end{aligned}
$$

On the other hand, a two-fold application of Zimmermann's formula relating normal products of different degrees (see Appendix) gives

$$
\Delta_{0}^{2} \Gamma^{(N)}=\left[\left(\Delta_{1}+r \Delta_{2}+s \Delta_{3}\right)^{2}+t \Delta_{1}+u \Delta_{2}+v \Delta_{3}\right] \Gamma^{(N)}
$$

where

$$
\begin{aligned}
& t=-i \Delta_{0}^{2} \Gamma^{(2)}(0,0), \\
& u=-i\left[\frac{d}{d p^{2}} \Delta_{0}^{2} \Gamma^{(2)}(p,-p)\right]_{p=0}, \\
& v=-i \Delta_{0}^{2} \Gamma^{(4)}(0,0,0,0) .
\end{aligned}
$$

Thus

$$
\begin{aligned}
& {\left[m^{2} \frac{\partial}{\partial m^{2}}+\mu^{2} \frac{\partial}{\partial \mu^{2}}+\beta \frac{\partial}{\partial g}-(N-2) \gamma\right] \Delta_{0} \Gamma^{(N)}} \\
& =\alpha m^{2}\left[\Delta_{0}^{2} \Gamma^{(N)}-t \Delta_{1}-u \Delta_{2}-v \Delta_{3}\right] \Gamma^{(N)} \\
& +\left[\left(m^{2} \frac{\partial r}{\partial m^{2}}+\mu^{2} \frac{\partial r}{\partial \mu^{2}}+\beta \frac{\partial r}{\partial g}\right) \Delta_{2}+\left(m^{2} \frac{\partial s}{\partial m^{2}}+\mu^{2} \frac{\partial s}{\partial \mu^{2}}+\beta \frac{\partial s}{\partial g}-2 \gamma s\right) \Delta_{3}\right] \Delta^{(N)} .
\end{aligned}
$$

If we now go to the region of large momenta, $p_{i}=\lambda r_{i}, \lambda \rightarrow \infty$, power counting $[12,13]$ gives

$$
\frac{\Delta_{0} \Gamma^{(N)}}{\Gamma^{(N)}} \sim \lambda^{-2} \log ^{a} \lambda, \quad \frac{\Delta_{0}^{2} \Gamma^{(N)}}{\Gamma^{(N)}} \sim \lambda^{-2} \log ^{b} \lambda .
$$

This implies that the linear terms in (4.6) must be proportional to $\Delta_{0} \Gamma^{(N)}$, so that we obtain, finally,

$$
\begin{aligned}
& {\left[m^{2} \frac{\partial}{\partial m^{2}}+\mu^{2} \frac{\partial}{\partial \mu^{2}}+\beta \frac{\partial}{\partial g}-(N-2) \gamma-\alpha m^{2} t\right]\left[\alpha m^{2}\right]^{-1}} \\
& \cdot\left[m^{2} \frac{\partial}{\partial m^{2}}+\mu^{2} \frac{\partial}{\partial \mu^{2}}+\beta \frac{\partial}{\partial g}-N \gamma\right] \Gamma^{(N)}=\alpha m^{2} \Delta_{0}^{2} \Gamma^{(N)} .
\end{aligned}
$$


For $N=2$ this result has been derived by Symanzik [13] by other methods. In order to apply Eq. (4.7) in short-distance analysis, further knowledge of the inhomogeneous term is required ${ }^{1}$. The reader is referred to Ref. [13] for an appropriate treatment of this problem.

\section{Appendix}

Derivation of the Expansion Formulas for $\Delta_{0} \Gamma^{(N)}$ and $\Delta_{0}^{2} \Gamma^{(N)}$

We wish to express $\Delta_{0} \Gamma^{(N)}$ and $\Delta_{0}^{2} \Gamma^{(N)}$ (defined in Sections III A and IV) in terms of the differential vertex operations $\Delta_{i}$ and $\Delta_{i} \Delta_{j}, i, j=1,2,3$. The expansions are based on a useful formula of Zimmermann [5].

Suppose $I_{G}$ is the unrenormalized integrand corresponding to a Feynman diagram $G$ containing a special vertex $V$. Suppose further that $R_{\phi}$ and $R_{\delta}, \phi<\delta$, are two alternative renormalized integrands derived from $I_{G}$ by the prescription [5]

$$
R_{\chi}=\sum_{U \in \mathscr{F}} \prod_{\gamma \in U}\left(-t_{\chi}^{\gamma}\right) I_{G}, \quad \chi=\phi, \delta,
$$

where $\mathscr{F}$ is the set of "forests" (families of nonoverlapping renormalization parts of $G$ ) and $t_{\chi}^{\gamma}$ is the Taylor operator on the external momenta of $\gamma$ with degree determined by $\chi$. The only difference between $R_{\phi}$ and $R_{\delta}$ is that in calculating the former the special vertex $V$ is assigned degree $\phi$, whereas for the latter $V$ has degree $\delta$. For all other vertices the degrees are identical in the two cases. Then

$$
R_{\phi}=R_{\delta}+\sum_{\tau} \sum_{U \in M(\tau)} \prod_{\gamma \in U}\left(-t_{\delta}^{\gamma}\right)\left(t_{\delta}^{\tau}-t_{\phi}^{\tau}\right) \sum_{U^{\prime} \in m(\tau)} \prod_{\gamma^{\prime} \in U^{\prime}}\left(-t_{\phi}^{\gamma^{\prime}}\right) I_{G}
$$

where the first sum is over all renormalization parts containing $V, M(\tau)$ is the set of all forests whose elements either contain $\tau$ properly or are disjoint from $\tau$, and $m(\tau)$ is the set of all forests whose elements are properly contained in $\tau$.

In the case of $\Delta_{0} \Gamma^{(N)}$, Eq. (A.2) with $\phi=2, \delta=4$, summed over all relevant Feynman diagrams, gives

$$
\Delta_{0} \Gamma^{(N)}=\left(\Delta_{1}+r \Delta_{2}+s \Delta_{3}\right) \Gamma^{(N)}
$$

where use has been made of Lorentz invariance to eliminate $D V O$ 's with only one derivative and to contract those with two derivatives. The coefficients may be deduced from (A.2) or, more quickly, from the

${ }^{1}$ The author is indebted to Symanzik for calling this to his attention. 
normalization conditions

$$
\begin{aligned}
\Delta_{1} \Gamma^{(2)}(0,0) & =\left.\frac{d}{d p^{3}} \Delta_{2} \Gamma^{(2)}(p,-p)\right|_{p=0}=\Delta_{3} \Gamma^{(4)}(0,0,0,0)=i \\
\Delta_{2} \Gamma^{(2)}(0,0) & =\Delta_{3} \Gamma^{(2)}(0,0)=\Delta_{1} \Gamma^{(4)}(0,0,0,0)=\Delta_{2} \Gamma^{(4)}(0,0,0,0) \\
& =\left.\frac{d}{d p^{2}} \Delta_{1} \Gamma^{(2)}(p,-p)\right|_{p=0}=\left.\frac{d}{d p^{2}} \Delta_{3} \Gamma^{(2)}(p,-p)\right|_{p=0}=0 .
\end{aligned}
$$

From (A.3) and (A.4) one obtains

$$
\begin{aligned}
& r=-\left.i \frac{d}{d p^{2}} \Delta_{0} \Gamma^{(2)}(p,-p)\right|_{p=0} \\
& s=-i \Delta_{0} \Gamma^{(4)}(0,0,0,0) .
\end{aligned}
$$

By similar reasoning we have in the case of two special vertices, $V_{1}$ and $V_{2}$, with $\phi_{1}=2, \phi_{2}=\delta_{1}=\delta_{2}=4$ (since only $V_{1}$ changes its degree, Zimmermann's formula (A.2) is still applicable),

$$
\Delta_{0} \Delta_{i} \Gamma^{(N)}=\left[\left(\Delta_{1}+r \Delta_{2}+s \Delta_{3}\right) \Delta_{i}+u_{i} \Delta_{2}+v_{i} \Delta_{3}\right] \Gamma^{(N)}, i=1,2,3
$$

with

$$
\begin{aligned}
& u_{i}=-\left.i \frac{d}{d p^{2}} \Delta_{0} \Delta_{i} \Gamma^{(2)}(p,-p)\right|_{p=0}, \\
& v_{i}=-i \Delta_{0} \Delta_{i} \Gamma^{(4)}(0,0,0,0) .
\end{aligned}
$$

Here the second and third quadratic terms on the righthand side arise from the cases $V_{1} \in \tau, V_{2} \notin \tau(\tau$ as in (A.2)), whereas the linear terms correspond to $V_{1} \in \tau, V_{2} \in \tau$.

Proceeding one step further, we may now treat the case $\phi_{1}=\phi_{2}=\delta_{2}=2$, $\delta_{1}=4$ :

$$
\begin{aligned}
\Delta_{0}^{2} \Gamma^{(N)} & =\left[\left(\Delta_{1}+r \Delta_{2}+s \Delta_{3}\right) \Delta_{0}+t \Delta_{1}\right] \Gamma^{(N)} \\
& =\left[\left(\Delta_{1}+r \Delta_{2}+s \Delta_{3}\right)^{2}+t \Delta_{1}+u \Delta_{2}+v \Delta_{3}\right] \Gamma^{(N)}
\end{aligned}
$$

where the term $t \Delta_{1} \Gamma^{(N)}$ comes from renormalization parts $\tau$ in (A.2) which contain both special vertices. Again the normalization conditions may be applied to give

$$
\begin{aligned}
& t=-i \Delta_{0}^{2} \Gamma^{(2)}(0,0) \\
& u=-\left.i \frac{d}{d p^{2}} \Delta_{0}^{2} \Gamma^{(2)}(p,-p)\right|_{p=0} \\
& v=-i \Delta_{0}^{2} \Gamma^{(4)}(0,0,0,0) .
\end{aligned}
$$

Acknowledgments. The author wishes to thank B. Schroer and R. Seiler for helpful discussions. 


\section{References}

1. Bogoliubov, N.N., Shirkov,D.W.: Introduction to the theory of quantized fields, New York: Interscience Publishers 1959.

2. Coleman, S., Jackiw, R.: Canonical and non-canonical scale symmetry breaking, M. I. T. report (1970).

3. Callan, Jr., C. G.: Phys. Rev. D2, 1541 (1970).

4. Symanzik, K.: Commun. math. Phys. 18, 227 (1970).

5. Zimmermann, W.: Lectures on elementary particles and quantum field theory, Vol. 1, p. 397, Cambridge: M. I. T. Press 1970.

6. Gell-Mann, M., Low, F.: Phys. Rev. 95, 1300 (1954).

7. - - Phys. Rev. 84, 350 (1951).

8. Courant, R., Hilbert, D.: Methods of mathematical physics, Vol. II, New York: Interscience Publishers 1953.

9. Lowenstein, J.: Univ. of Pittsburgh report NYO-3829-67 (1971).

10. Symanzik, K.: Commun. math. Phys. 16, 48 (1970).

11. Wilson, K.: Phys. Rev. 179, 1499 (1969).

12. Weinberg, S.: Phys. Rev. 118, 838 (1960).

13. Symanzik, K.: Small-distance-behaviour analysis and Wilson expansions, DESY report (1971).

J. H. Lowenstein

Dept. of Physics

University of Pittsburgh

Pittsburgh, Pennsylvania 15213, USA 